\title{
Click Chemistry (CuAAC) and Detection of Tagged de novo Synthesized Proteins in Drosophila
}

Kathrin Marter ${ }^{1,2}$, Oliver Kobler ${ }^{4}$, Ines Erdmann ${ }^{1,2}$, Elaheh Soleimanpour ${ }^{1}$, Peter Landgraf ${ }^{1,2}$, Anke Müller, ${ }^{1,2}$, Julia Abele ${ }^{1,2}$, Ulrich Thomas ${ }^{4, *}$ and Daniela C. Dieterich ${ }^{1,2,3, *}$

${ }^{1}$ Neuronal Plasticity and Communication, Institute for Pharmacology and Toxicology, Otto-vonGuericke-University Magdeburg, Magdeburg, Germany; ${ }^{2}$ Research Group Neuralomics, Leibniz Institute for Neurobiology, Magdeburg, Germany; ${ }^{3}$ Center for Behavioral Brain Sciences, Magdeburg, Germany; ${ }^{4}$ Department of Neurochemistry and Molecular Biology, Leibniz Institute for Neurobiology, Magdeburg, Germany

*For correspondence: daniela.dieterich@med.ovgu.de; thomas@lin-magdeburg.de

[Abstract] Copper-catalyzed azide-alkyne-cycloaddition (CuAAC), also known as 'click chemistry' serves as a technique for bio-orthogonal, that is, bio-compatible labeling of macromolecules including proteins or lipids. Click chemistry has been widely used to covalently, selectively, and efficiently attach probes such as fluorophores or biotin to small bio-orthogonal chemical reporter groups introduced into macromolecules. In bio-orthogonal non-çanonical amino acid tagging (BONCAT) and fluorescent noncanonical amino acid tagging (FUNCAT) proteins are metabolically labeled with a non-canonical, azidebearing amino acid and subsequently CuAAC-clicked either to an alkyne-bearing biotin (BONCAT) for protein purification, Western blot, or mass spectrometry analyses or to an alkyne-bearing fluorophore (FUNCAT) for immunohistochemistry. In combination with mass spectrometry, these kinds of labeling and tagging strategies are a suitable option to identify and characterize specific proteomes in living organisms without the need of prior cell sorting. Here, we provide detailed protocols for FUNCAT and BONCAT click chemistry and the detection of tagged de novo synthesized proteins in Drosophila melanogaster.

Keywords: Bio-orthogonal chemical reporters, Click chemistry, CuAAC, Protein tagging, Drosophila melanogaster, Proteomic profiling, Protein synthesis

[Background] Bio-orthogonal chemical reporters are used to visualize and identify experimentally defined entities of macromolecules in living cells and organisms. Originally developed as a means for protein tracking and thus complementary to tagging by fluorescent proteins (e.g., GFP) or antibodylabeling (Tsien, 1998; Massoud and Gambhir, 2003), they can also be used to monitor glycans, lipids, nucleic acids or virus particles (Wang et al., 2003; Kho et al., 2004; Seo et al., 2004; Laughlin et al., 2008; Martin et al., 2008; Martin and Cravatt, 2009; Palaniappan and Bertozzi, 2016, Nguyen et al., 2017). Azides and alkynes are particularly well suited as bio-orthogonal chemical reporters (Prescher and Bertozzi, 2005; Dieterich, 2010) because they typically do not exist in living systems, thus precluding unwanted side reactions during tagging. At the same time, and in contrast to radioactive reporters, they allow for highly specific reactions with established tags (e.g., fluorophores or affinity tags). 
The traditional copper-catalyzed azide-alkyne-cycloaddition (CuAAC [Rostovtsev et al., 2002; Tornoe et al., 2002], AAC [Huisgen, 1963]), widely known as 'click chemistry' is one of the principal bioorthogonal labeling reactions as it is fast, chemically highly selective, and applicable over a wide range of biochemically compatible buffers, $\mathrm{pH}$ and temperature. Azide groups and alkyne groups exhibit high reactivity towards each other, and covalent bonding between them manifests in the formation of a stable triazole ring. Azide-bearing molecules can be detected using alkyne-bearing tags and vice versa. Whereas, however, alkynes can only be CuAAC-clicked, azides can also be tagged by Staudinger ligation (Saxon and Bertozzi, 2000; Kiick et al., 2002) or strain-promoted cycloaddition (e.g., Agard et al., 2004; reviewed in Lallana et al., 2011), both with a slower reaction rate (to review chemical reactions in bio-orthogonal chemistry see Prescher and Bertozzi, (2005); Ramil and Lin, (2013).

In order to tag proteomes on the basis of click chemistry, we developed bio-orthogonal non-canonical amino acid tagging (BONCAT). In the prototypic, non-cell-type- specific BONCAT approach, the noncanonical amino acid azidohomoalanine (AHA) (or homopropargylglycine [HPG]) is used to compete with methionine for tRNA loading and translational incorporation using the endogenous protein synthesis machinery. AHA bears an azide 'handle' for alkyne-bearing biotin tagging, allowing for subsequent protein purification, Western blot or mass spectrometry analyses (Dieterich et al., 2006). Similarly, in fluorescent non-çanonical amino acid tagging (FUNCAT), AHA is clicked to an alkyne-bearing fluorophore for immunohistochemistry (Beatty et al., 2006; Dieterich et al., 2010). Most recently, this method was further advanced to enable the direct visualization of single newly synthesized proteins via proximity ligation assay (PLA) techniques (FUNCAT-PLA, tom Dieck et al., 2015). The expansion of the method to cell-type specific labeling with e.g., azidonorleucine (ANL) requires a mutation of the target organism to provide a cell-type specific MetRS capable to recognize ANL (first mutant MetRS capable to process ANL from E. Coli, Link et al., 2006, mMetRS ${ }^{L 274 G}$ in astrocytes, Müller et al., 2015; mMetRS ${ }^{\mathrm{L} 274 \mathrm{G}}$ and/or dMetRS ${ }^{\mathrm{L} 262 \mathrm{G}}$ in several Drosophila tissues (Erdmann et al., 2015; Niehues et al., 2015). ANL has a longer side chain than AHA and, therefore, does not fit the binding pocket of the endogenous MetRS. Critically, this approach does not require methionine depletion as the MetRS ${ }^{\text {LtoG }}$ has a higher affinity for ANL compared to AHA.

In the following, we provide detailed BONCAT, and FUNCAT protocols for cell-type specific metabolic labeling of de novo synthesized proteins with ANL in Drosophila according to Erdmann et al. (2015) and Niehues et al. (2015).

\section{Part I: Fluorescent non-canonical amino acid tagging (FUNCAT)-Protocol}

The procedure is described for preparations of larval body walls, which comprise various tissues and cell types including body wall and tracheal epithelia, sensory neurons, motor neurons, peripheral glia, and muscles. Additional tissues such as the CNS, imaginal discs or salivary glands may be left attached.

\section{Materials and Reagents}

\section{Tungsten needles}


2. Dish

3. Magnetic foil

4. Stainless steel pins

5. $1.5 \mathrm{ml}$ Eppendorf tubes

6. Pasteur pipettes (Carl Roth, catalog number: 4518.1)

7. 48-well plate (Sigma-Aldrich, catalog number: CLS3548)

8. Microscope slides (Carl Roth, catalog number: H868.1)

9. Cover slips (Carl Roth, catalog number: H874.2)

10. Dissection plate: wax plate (self-made, alternatively PDMS or magnetic plates) for dissection and fixation of larval body walls

11. Drosophila strains as described in Erdmann et al. (2015)

12. Ultra-pure water (Carl Roth, catalog number: AE72)

13. Dimethyl sulfoxide (DMSO, Sigma-Aldrich, catalog number: D8418)

14. $1 \mathrm{mM}$ tetramethylrhodamine- (TAMRA) alkyne-tag (Thermo Fisher Scientific, catalog number: T10183) in DMSO in $10 \mu$ aliquots, store at $-20^{\circ} \mathrm{C}$ for up to 1 year, avoid repeated freezing and thawing

15. $200 \mathrm{mM}$ triazole ligand (TBTA; Tris[(1-benzyl-1H-1,2,3-triazol-4-yl)methyl]amine) (SigmaAldrich, catalog number: 678937) in DMSO in $10 \mu \mathrm{l}$ aliquots, store at $-20^{\circ} \mathrm{C}$ for up to 1 year, avoid repeated freezing and thawing

16. 400 mM TCEP (Tris(2-carboxyethyl)phosphine hydrochloride) (Sigma-Aldrich, catalog number: C4706) in ultrapure water (prepare fresh before use)

17. $200 \mathrm{mM} \mathrm{CuSO}_{4}$ (Sigma-Aldrich, catalog number: 1027900250) in ultrapure water (prepare fresh before use)

18. VectaShield ${ }^{\mathrm{TM}}$ Mounting Medium (Fisher Scientific, catalog number: 13273694)

19. Transparent nail polish (drugstore)

20. $\mathrm{NaCl}$ (Carl Roth, catalog number: 3957)

21. $\mathrm{KCl}$ (Carl Roth, catalog number: 6781)

22. $\mathrm{MgCl}_{2} \cdot 6 \mathrm{H}_{2} \mathrm{O}$ (Carl Roth, catalog number: 2189)

23. $\mathrm{NaHCO}_{3}$ (Carl Roth, catalog number: 8551)

24. Sucrose (Carl Roth, catalog number: 4621)

25. Trehalose (Carl Roth, catalog number: 5151)

26. HEPES (Carl Roth, catalog number: 9105)

27. $\mathrm{CaCl}_{2}$ (Carl Roth, catalog number: T885)

28. $\mathrm{Na}_{2} \mathrm{HPO}_{4}$ (Carl Roth, catalog number: X987)

29. $\mathrm{NaH}_{2} \mathrm{PO}_{4}$ (Carl Roth, catalog number: T879)

30. PFA (Carl Roth, catalog number: 0335)

31. $\mathrm{KH}_{2} \mathrm{HPO}_{4}$ (Carl Roth, Germany, catalog number: 3904)

32. $\mathrm{NaOH}$ (Carl Roth, catalog number: 6771)

33. $\mathrm{HCl}$ (Carl Roth, Germany, catalog number: 9277) 
34. $20 \%$ Triton $^{\circledR}$ X-100 (Carl Roth, catalog number: 3051 )

35. Tween ${ }^{\circledR}-20$ (Carl Roth, catalog number: 9127 )

36. Hemolymph-like solution, HL3 (see Recipes)

37. $4 \%$ paraformaldehyde (PFA), (see Recipes)

38. Phosphate buffer (PB), pH 7.2 (see Recipes)

39. $1 \times$ PBS pH 7.4 or 7.8 (see Recipes)

40. PBT (see Recipes)

41. PBS-Tw (see Recipes)

42. 50x Protease Inhibitor (PI) w/o EDTA (see Recipes)

43. Homogenization buffer (see Recipes)

44. $20 \%$ (w/v) SDS (see Recipes)

45. PBS-SDS pH 7.8 (see Recipes)

46. PBS-Igepal-630 (see Recipes)

47. 2x SDS elution buffer (see Recipes)

\section{Equipment}

1. Binocular (e.g., Leica, model: $\mathrm{MZ10F}$ )

2. Forceps (Fine Science Tools, catalog number: 11255-20)

3. Scissors (Fine Science Tools, catalog number: 15002-08)

4. Reciprocating shaker (e.g., GFL, model 3006)

5. Vortex mixer (e.g., Scientific Industries, model: Vortex-Genie 1 or 2)

6. $4{ }^{\circ} \mathrm{C}$ refrigerator

7. $-20^{\circ} \mathrm{C}$ freezer

\section{Procedure}

Larvae expressing the dMetRS ${ }^{\mathrm{L} 262 \mathrm{G}}$ cell-type specifically and being fed with the non-canonical amino acid azidonorleucine (ANL) are needed. A detailed protocol on cell-type specific metabolic labeling of proteins with azidonorleucine in Drosophila is available (Erdmann et al., 2017).

1. Dissect larval body walls according to previously reported procedures by Brent et al. (2009) or Ramachandran and Budnik (2010). A detailed Video for larval body wall dissections is provided here (Video 1). Figure 1 shows the necessary materials and tools for larval body dissection. In brief, immobilize and gently stretch a rinsed and still, wet larva dorsal side up using needles positioned at short distance $(\sim 0.2 \mathrm{~mm})$ from the anterior and posterior ends on a dissection plate. Cover the animal with HL3 solution (see Recipes). With scissor blades held vertically and moderately pressed onto the larval cuticle about 0.5 to $1 \mathrm{~mm}$ from the posterior end, generate a hole with a single cut. From this site and now holding the blades in horizontal orientation cut the larva along its dorsal midline towards either of the two needles. Thereby avoid that the lower 
(inside) blade of the scissor scratches the ventral body wall. Make short cuts to the right and the left at either end. Push one end such that stretching is released and inner organs such as the gut and fat body are squeezed out. Using forceps, remove organs that are irrelevant for further analysis. Use additional needles to unfold and span the body wall symmetrically (i.e., flatten the body wall), avoiding overstretching.

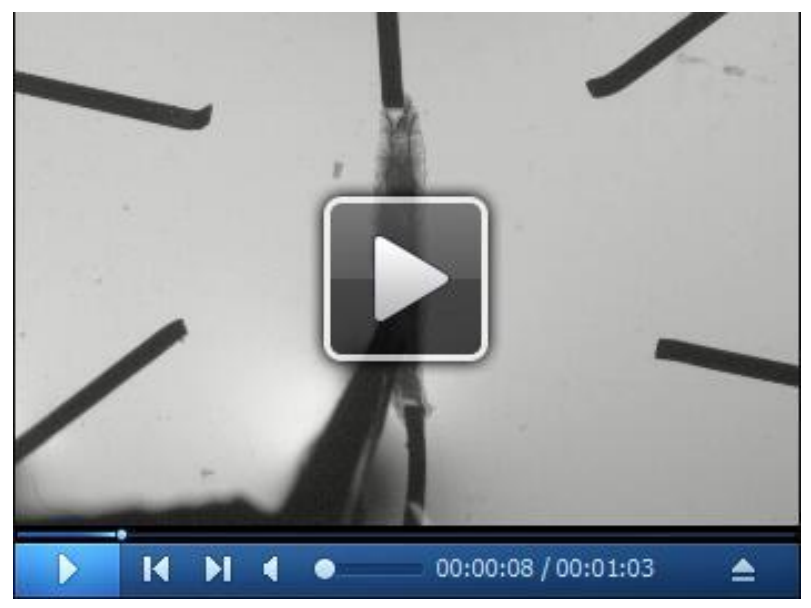

Video 1. Larval body wall dissection at 3-fold time-lapse. A wet larva is placed on the dissection chamber shown in the supplementary figure. Using the central, straight clips the animal is immobilized and moderately stretched, dorsal side up. The animal is then covered with $\mathrm{Ca}^{2+}$-free saline. Using the spring scissors a hole is squeezed in at about $75 \%$ length, serving as a starting point for cutting along the dorsal midline, first direction mouth hook and then towards the posterior end. Short incisions are made to the right and the left at either end. To avoid scratching the body wall muscles and harming the larval brain with the blades, the scissors are slightly lifted during the cutting. Once cut, the larva is pushed a bit to squeeze out inner organs such as the gut and fat body and thus to ease their removal. The lateral clips are then used to carefully span the body wall.

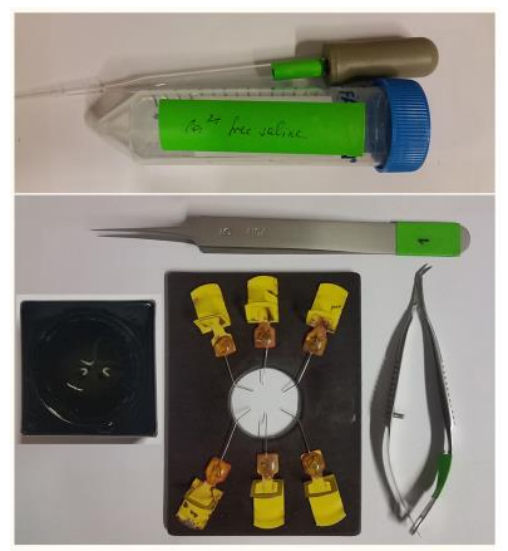

Figure 1. Materials and tools for larval body wall dissection. Include $\mathrm{Ca}^{2+}$-free saline, a tap water- or saline-containing dish for rinsing larvae prior to dissection, fine forceps (e.g., Dumont \#5) and spring scissors (e.g., Vannas-type, FST 15002-08). As an alternative to the widely used 
combination of PDMS plates with sharp tungsten needles, dissections can be carried out on a chamber $\left(\sim 5 \times 8 \mathrm{~cm}^{2}\right)$ that is based on a magnetic foil with a central hole of about $2 \mathrm{~cm}$ diameter, glued to a 1 to $2 \mathrm{~mm}$ thick glass slide. Stainless steel pins glued to iron-based holders and with sharp, hook-like endings are used as movable clips.

2. Pre-fix prepared body walls for about $30 \mathrm{~s}$ by adding $2-3$ drops of $4 \%$ PFA (see Recipes) with a Pasteur pipette. Avoid to drop the solution directly on the body wall section to prevent seizurelike contractions; 1 to $2 \mathrm{~cm}$ in distance is fine.

3. Remove the HL3-PFA solution and replace it by 4\% PFA, transfer the plate into a humid and dark box and fix for $20 \mathrm{~min}$. During fixation and all subsequent steps keep samples protected from light, e.g., in a dark and humid box with the lid closed to avoid evaporation of the fixative and subsequent solutions and to protect the EGFP fluorescence from fading. All washing steps are performed at room temperature (RT) under gentle agitation on a reciprocating shaker (25 rounds per minute).

4. Wash the body walls three times with PBT (see Recipes) for 15 min each.

5. Wash the body walls three times with $1 \times \mathrm{PBS}$ pH 7.8 (see Recipes) for $15 \mathrm{~min}$ each.

6. Place the fixed body walls in a 48-well plate in $200 \mu \mathrm{l} 1 \mathrm{x} \mathrm{PBS} \mathrm{pH} \mathrm{7.8;} \mathrm{put} \mathrm{the} \mathrm{48-well} \mathrm{plate} \mathrm{into}$ a dark and humid box with lid closed until the FUNCAT reaction mix (see next step) is ready (about $5 \mathrm{~min}$ ) at RT.

7. Prepare the FUNCAT-reagent at $\mathrm{RT}$ in a $1.5 \mathrm{ml}$ reaction tube: to $1 \mathrm{ml} 1 \mathrm{x} \mathrm{PBS} \mathrm{pH} 7.8$ add:

a. $1 \mu \mathrm{l} 200 \mathrm{mM}$ triazole ligand (final concentration $200 \mu \mathrm{M}$ ).

b. $0.2 \mu \mathrm{l} 1 \mathrm{mM}$ TAMRA-alkyne-tag (final concentration $0.2 \mu \mathrm{M}$ ).

c. $1 \mu \mathrm{l} 400 \mathrm{mM}$ TCEP solution (final concentration $400 \mu \mathrm{M}$ ).

d. After each addition step, vigorously mix for $10 \mathrm{~s}$ using a vortex mixer at the highest possible speed; we obtain best results at 2,700 rpm.

e. Continue adding $1 \mu \mathrm{l} 200 \mathrm{mM} \mathrm{CuSO}_{4}$ (final concentration $200 \mu \mathrm{M}$ ).

f. Mix for $30 \mathrm{~s}$ using a vortex mixer at highest possible speed (2,700 rpm).

g. Keep FUNCAT reaction mix ready at RT until use; quickly proceed with Step 8.

8. After removing the $1 \times \mathrm{PBS} \mathrm{pH} 7.8$ from the body walls in the 48-well plate, immediately apply $200 \mu \mathrm{l}$ FUNCAT-reagent to the body walls.

9. Incubate overnight at $4{ }^{\circ} \mathrm{C}$ under gentle agitation on a reciprocating shaker.

10. Remove FUNCAT-reagent and wash body walls three times with $200 \mu \mathrm{l}$ PBS-Tw (see Recipes) for 15 min each.

11. Wash body walls three times with $200 \mu \mathrm{I}$ PBT for 15 min each.

12. For co-staining with antibodies follow standard protocols. This type of preparation usually does not require blocking steps. In brief, dilute primary antibodies in PBT to appropriate concentrations and incubate samples overnight at $4{ }^{\circ} \mathrm{C}$ or for $1 \mathrm{~h}$ at room temperature. After washing three times with $200 \mu \mathrm{l}$ PBT for 15 min each apply fluorescently-labeled secondary antibodies, typically at concentrations of 1:300 (if for instance purchased from Jackson 
Laboratories) in PBT and incubate samples overnight at $4{ }^{\circ} \mathrm{C}$ or for $1 \mathrm{~h}$ at room temperature. Note that red fluorescent dyes such as Сy3 or Alexa 568 are not useful here as their excitation and emission spectra largely overlap with that of TAMRA.

13. Arrange body walls on microscope slides; mount them with a few drops of VectaShield ${ }^{\mathrm{TM}}$ Mounting Medium and gently place a cover slip on the body walls.

14. Seal the edges of the cover slips with a thin layer of clear nail polish.

15. Store the slides at $4{ }^{\circ} \mathrm{C}$ until imaging and at $-20^{\circ} \mathrm{C}$ for long term storage. An example for a FUNCAT staining of de novo synthesized glial proteins is provided in Figure 2.

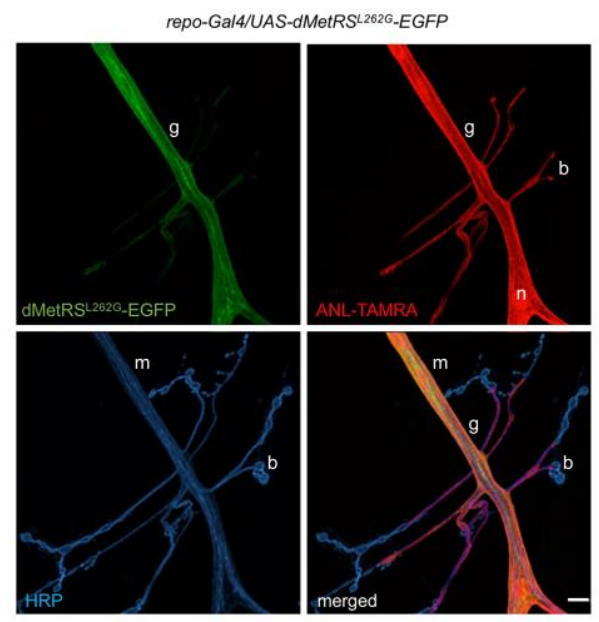

Figure 2. Cell-type-specific in situ labeling of glial proteins via FUNCAT. ANL incorporation into larval proteins is monitored via FUNCAT on targeted expression of dMetRSL262G-EGFP in glia cells (repo-Gal4/UAS-dMetRS ${ }^{L 262 G}$-EGFP) at larval neuromuscular junctions (muscles 6/7, segment A2). Co-staining with the neuron-specific marker anti-HRP reveals that, wherever nerve terminal boutons $(\mathrm{b})$ of motor neurons $(\mathrm{m})$ and glial protrusions $(\mathrm{g})$ are in close contact, ANL-TAMRA signal is indeed restricted to the dMetRSL262G-EGFP-expressing glial cell. Notably, dMetRS ${ }^{\mathrm{L} 262 \mathrm{G}}$-EGFP is predominantly found in the cytosol, whereas TAMRA-harboring proteins are detected throughout the glia cell including nuclei $(\mathrm{n})$ and glial protrusions $(\mathrm{g})$ in close proximity to the first synaptic boutons. Scale bar is $5 \mu \mathrm{m}$.

\section{Part II: Bio-orthogonal non-canonical amino acid tagging (BONCAT)-Protocol}

\section{Materials and Reagents}

1. Syringe

2. Zeba Desalt Spin Columns (Thermo Scientific, catalog number: 10627354)

3. Dissection plate: wax plate (self-made, alternatively PDMS or magnetic plates) for dissection and fixation of larval body walls

4. $1.5 \mathrm{ml}$ tubes (e.g., Eppendorf, catalog number: 0030120086)

5. $15 \mathrm{ml}$ centrifuge tubes (e.g., Greiner bio-one, catalog number: 188261) 
6. Drosophila

7. Liquid nitrogen

8. $\mathrm{CO}_{2}$

9. Ultra-pure water (Carl Roth, catalog number: AE72)

10. Benzonase ${ }^{\circledR}$ Nuclease (Sigma-Aldrich, catalog number: E1014)

11. $25 \mathrm{mM}$ Biotin- $\mathrm{PEO}_{3}$-alkyne-Tag in 1x PBS pH 7.8 (synthesized according to Link and Tirrell, [2003]) in $10 \mu \mathrm{l}$ aliquots, store at $-20^{\circ} \mathrm{C}$ for up to 1 year, avoid repeated freezing and thawing

12. Copper(I) Bromide (Acros Organics, catalog number: 199582500)

13. $200 \mathrm{mM}$ triazole ligand (TBTA; Tris[(1-benzyl-1H-1,2,3-triazol-4-yl)methyl]amine) (SigmaAldrich, catalog number: 678937) in DMSO in $10 \mu \mathrm{l}$ aliquots, store at $-20^{\circ} \mathrm{C}$ for up to 1 year, avoid repeated freezing and thawing

14. NeutrAvidin ${ }^{T M}$ agarose (Thermo Scientific, catalog number: 29204)

15. $\mathrm{NaCl}$ (Carl Roth, catalog number: 3957)

16. $\mathrm{KCl}$ (Carl Roth, catalog number: 6781)

17. $\mathrm{MgCl}_{2} \cdot 6 \mathrm{H}_{2} \mathrm{O}$ (Carl Roth, catalog number: 2189)

18. $\mathrm{NaHCO}_{3}$ (Carl Roth, catalog number: 8551)

19. Sucrose (Carl Roth, catalog number: 4621)

20. Trehalose (Carl Roth, catalog number: 5151)

21. HEPES (Carl Roth, catalog number: 9105)

22. $\mathrm{CaCl}_{2}$ (Carl Roth, catalog number: T885)

23. Roche Complete EDTA-free Protease Inhibitor Cocktail Tablet (Sigma-Aldrich, catalog number: 04693132001)

24. SDS (Carl Roth, catalog number: 2326.3)

25. Igepal-630 (Sigma-Aldrich, catalog number: 18896)

26. Tris, pH 6.8 (SERVA, catalog number: 37180 )

27. $\beta$-mercaptoethanol (Sigma-Aldrich, catalog number: 63689)

28. Glycerol (Carl Roth, catalog number: 3783)

29. Bromophenol blue (Carl Roth, catalog number: A512)

30. $20 \%$ Triton ${ }^{\circledR}$ X-100 (Carl Roth, catalog number: 3051 )

31. Nitrocellulose membranes (e.g., Protran BA85, $0.45 \mu \mathrm{m}$, Whatman)

32. Hemolymph-like solution, HL3 (see Recipes)

33. 50x Protease Inhibitor (PI) w/o EDTA (see Recipes)

34. Homogenization buffer (see Recipes)

35. $20 \%(w / v)$ SDS (see Recipes)

36. PBS-SDS pH 7.8 (see Recipes)

37. PBS-Igepal-630 (see Recipes)

38. $2 x$ SDS elution buffer (see Recipes) 


\section{Equipment}

1. Pipette

2. Binocular (e.g., Leica, model: MZ10F)

3. Forceps (Fine Science Tools, catalog number: 11255-20)

4. Scissors (Fine Science Tools, catalog number: 15002-08)

5. Glass homogenizers (Wheaton, via Fisher Scientific, catalog number: 10449651)

6. Rotator/end over end mixer (e.g., neoLab, catalog number: 2-1175)

7. Mini centrifuge (e.g., neoLab, model: Spectrafuge 3-1810)

8. Micro centrifuge (e.g., Eppendorf, model: 5424)

9. Vortex mixer (e.g., Scientific Industries Inc., model: Vortex-Genie 2)

10. Heating block (e.g., VWR, catalog number: 12621-100)

11. Centrifuge (Eppendorf, catalog number: $5810 \mathrm{R}$ )

12. Hoefer Mighty Small System SE250 (Amersham Biosciences)

13. $-20^{\circ} \mathrm{C}$ freezer

14. $-80^{\circ} \mathrm{C}$ freezer

\section{Procedure}

\section{A. Preparation of material}

This protocol was established for the lysis of either 8 larval body walls, 50 laval brains or 20 fly heads to a final volume of $500 \mu$ l. Larvae or flies expressing the dMetRS ${ }^{\text {L262G }}$ cell-type specifically and that were fed with the non-canonical amino acid azidonorleucine (ANL), to enable its incorporation into proteins, are needed. A detailed protocol on cell-type specific metabolic labeling of proteins with ANL in Drosophila is available (Erdmann et al., 2017).

1. Preparation of larval body walls

a. Prepare larval body walls in HL3 solution (see Recipes) as described above in the Procedure of Part I according to previously reported procedures by Brent et al. (2009) or Ramachandran and Budnik (2010). However, skip the final flattening necessary for FUNCAT and immunostaining (Step 1 in the Procedure of Part I).

b. Cut the most anterior and posterior parts to remove the mouth hook and the posterior spiracles from the body wall.

c. Take the remaining body wall using forceps and wipe it carefully over a tissue to remove excess HL3 solution.

d. Transfer the body wall directly into a glass homogenizer containing $50 \mu \mathrm{l}$ homogenization buffer (see Recipes) on ice and repeat dissections until 8 body walls per group are collected. Alternatively, larval body walls may be processed at a later time point. To do so, collect 8 body walls in $50 \mu \mathrm{l}$ homogenization buffer in a $1.5 \mathrm{ml}$ tube on ice, shock-freeze in liquid 
nitrogen and store at $-80^{\circ} \mathrm{C}$ until further processing. To continue, thaw frozen samples on ice before proceeding with Step B.

2. Preparation of larval brains

a. Prepare larval brains in HL3 solution by opening the dorsal site of a Drosophila larva and remove the brains from the heads using forceps.

b. Wipe the larval brain carefully over a tissue to remove excess HL3 solution and transfer it into a glass homogenizer with $50 \mu$ homogenization buffer on ice and repeat dissections until 50 brains per group are collected. Alternatively, larval brains may be processed at a later time point. To do so, collect 50 brains in $50 \mu \mathrm{l}$ homogenization buffer in a $1.5 \mathrm{ml}$ tube on ice, shock-freeze in liquid nitrogen and store at $-80{ }^{\circ} \mathrm{C}$ until further processing. To continue, thaw frozen samples on ice before proceeding with Step B.

3. Preparation of fly heads

a. Anesthetize flies with $\mathrm{CO}_{2}$.

b. Cut off 20 fly heads using either a pair of small scissors or forceps and transfer them directly into a glass homogenizer containing $50 \mu$ homogenization buffer (see Recipes) as shown in Video 2. Figure 3 shows the main dissection steps. Alternatively, fly heads walls may be processed at a later time point. To do so, collect heads in $50 \mu$ homogenization buffer in a $1.5 \mathrm{ml}$ tube on ice, shock-freeze in liquid nitrogen and store at $-80^{\circ} \mathrm{C}$ until further processing. To continue, thaw frozen samples on ice before proceeding with Step B.

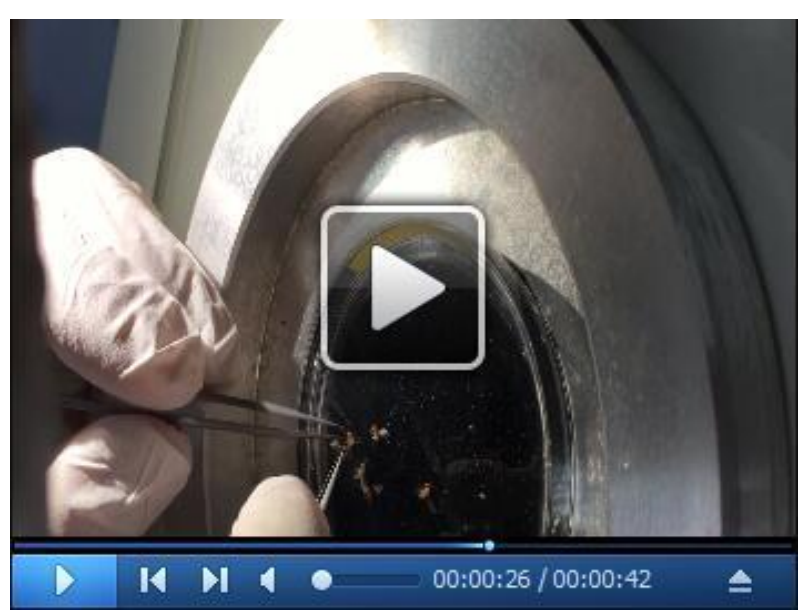

Video 2. Fly head dissection. First, a glass homogenizer containing $50 \mu$ homogenization buffer is put on ice. Flies are anesthetized with $\mathrm{CO}_{2}$ in the vial for a few seconds. Once the flies are immobile, they are transferred to a PDMS dissection plate, which is placed on top of a cold plate. Using a pair of fine forceps the abdomen is hold, and the head is gently pinched off with a second pair of forceps or fine scissors. Finally, the head is directly transferred into the glass homogenizer containing homogenization buffer. Continue until a total of 20 dissected heads are collected in the homogenizer. 


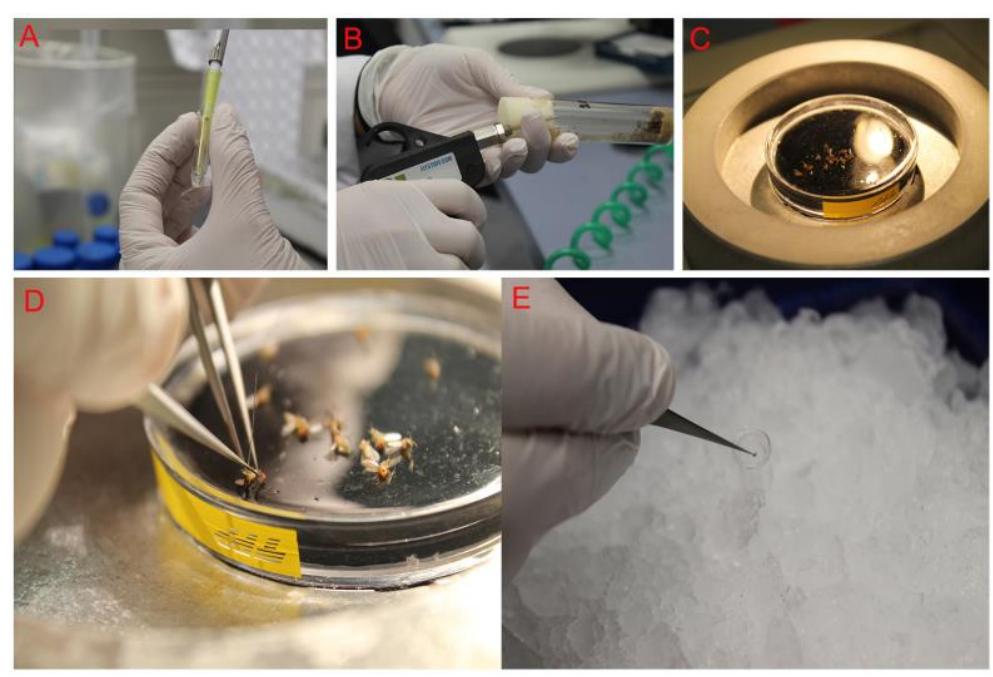

Figure 3. Fly head dissection. A. Prepare a glass homogenizer containing $50 \mu \mathrm{l}$ homogenization buffer, put it on ice. B. Anesthetize flies with $\mathrm{CO}_{2}$. C. Transfer anesthetized flies to a PDMS dissection plate, which is placed on top of a cold plate. D. Use fine forceps to keep hold of the abdomen and a second pair of forceps or fine scissor to gently pinch off/cut off the fly head. E. Transfer the head directly into the glass homogenizer containing homogenization buffer. Repeat steps (Figures 3D and 3E) for a total of 20 dissected heads.

B. Protein extraction

Note: If not stated otherwise all subsequent steps are performed on ice.

1. Optionally (for frozen and stored material): Transfer the thawed samples into a glass homogenizer containing $50 \mu \mathrm{l}$ homogenization buffer.

2. Homogenize material with the homogenization pistil for $60-120 \mathrm{~s}$ or until the suspension is homogeneous and devoid of any debris on ice.

3. Add $2.5 \mu \mathrm{l} 20 \%$ SDS (see Recipes) and $1 \mu \mathrm{l}$ Benzonase $^{\circledR}$ and homogenize again.

4. Spin the material down with a mini centrifuge to collect all of the samples at the bottom of the tube (approximately 2,000 $\times \mathrm{g}$ for $5 \mathrm{~s}$ ) at RT.

5. Transfer the suspension into a $1.5 \mathrm{ml}$ tube.

6. Rinse the glass homogenizer with $50 \mu \mathrm{l}$ homogenization buffer, spin the material down with the mini centrifuge (approximately 2,000 $\times \mathrm{g}$ for $5 \mathrm{~s}$ ) and transfer the suspension into the $1.5 \mathrm{ml}$ tube from Step B5.

7. Incubate suspension for $20 \mathrm{~min}$ at room temperature in an end over end mixer.

8. Spin the material down with the mini centrifuge.

9. Heat suspension for $7 \mathrm{~min}$ at $95^{\circ} \mathrm{C}$ and subsequently cool it down on ice for $5 \mathrm{~min}$.

10. Add $5 \mu \mathrm{l} 20 \%$ Triton $^{\circledR} X-100$ to the suspension and mix briefly using a vortex mixer.

Note: Suspension should be cooled down completely; otherwise the proteins will denature when adding $20 \%$ Triton $^{\circledR} X-100$.

11. Add $391.5 \mu \mathrm{l}$ homogenization buffer to a final volume of approximately $500 \mu \mathrm{l}$.

12. Incubate suspension for $1 \mathrm{~h}$, at $4{ }^{\circ} \mathrm{C}$ in an end over end mixer. 
13. Centrifuge at $3,000 \times \mathrm{g}$ for $5 \mathrm{~min}$ at $4{ }^{\circ} \mathrm{C}$ and transfer supernatant (= protein lysate) into a new $1.5 \mathrm{ml}$ tube sitting on ice.

\section{Click chemistry}

Note: This protocol was modified according to Dieterich et al. (2007). In contrast to the FUNCAT reaction reagent, the individual components of the click chemistry are directly added to the protein lysates.

1. Weigh $\mathrm{Cu}(\mathrm{l}) \mathrm{Br}$ into several $2 \mathrm{ml}$ tubes and calculate the amount of ultra-pure water needed to reach a concentration of $10 \mathrm{mg} / \mathrm{ml}$ for each tube. Do not dissolve the $\mathrm{Cu}(\mathrm{I}) \mathrm{Br}$ yet. One $\mathrm{Cu}(\mathrm{I}) \mathrm{Br}$ suspension can be used for two click reactions.

2. Add $0.5 \mu \mathrm{l}$ of $200 \mathrm{mM}$ triazole-ligand (final concentration $200 \mu \mathrm{M}$ ) to the protein lysate and mix for $10 \mathrm{~s}$ using a vortex mixer at highest speed, 2,700 rpm.

3. Add $0.5 \mu \mathrm{l}$ of $25 \mathrm{mM}$ Biotin-PEO 3 -alkyne tag (final concentration $25 \mu \mathrm{M}$ ) to the protein lysate and mix for $10 \mathrm{~s}$ using a vortex mixer at highest speed, 2,700 rpm.

4. Add the appropriate amount of ultra-pure water to your $\mathrm{Cu}(\mathrm{l}) \mathrm{Br}(10 \mathrm{mg} / \mathrm{ml})$ and $\mathrm{mix}$ for $10 \mathrm{~s}$ using a vortex mixer at highest speed, 2,700 rpm.

5. Add $10 \mu \mathrm{l}$ of the $10 \mathrm{mg} / \mathrm{ml} \mathrm{Cu}(\mathrm{l}) \mathrm{Br}$ suspension (final concentration $0.5 \mathrm{mg} / \mathrm{ml}$ ) to your protein lysate and mix for $30 \mathrm{~s}$ using a vortex mixer at highest speed, 2,700 rpm.

6. Incubate the reaction mix overnight at $4^{\circ} \mathrm{C}$ in an end over end mixer.

7. Centrifuge the reaction mix at $3,000 \times g$ for 5 min at $4{ }^{\circ} \mathrm{C}$.

Note: The $\mathrm{Cu}(\mathrm{I}) \mathrm{Br}$ and remaining triazole-ligand will precipitate at the bottom of the tube.

8. Transfer the supernatant into a new $1.5 \mathrm{ml}$ tube.

D. Desalting

Note: All subsequent steps are performed on ice.

1. Remove the bottom's closure from the desalting column, loose cap and place the column in a $15 \mathrm{ml}$ centrifuge tube.

2. Centrifuge at $1,000 \times g$ for $2 \mathrm{~min}$ at $4{ }^{\circ} \mathrm{C}$.

3. Discard the flow-through after each centrifugation step of the washing procedure.

4. Add $1 \mathrm{ml}$ of PBS-SDS pH 7.8 (see Recipes) to the column.

5. Centrifuge at $1,000 \times g$ for $2 \mathrm{~min}$ at $4{ }^{\circ} \mathrm{C}$.

6. Repeat Steps D4 and D5 twice.

7. Place the column in a new $15 \mathrm{ml}$ centrifuge tube and add the clicked protein lysate to the column.

8. Centrifuge at $1,000 \times g$ for $2 \mathrm{~min}$ at $4{ }^{\circ} \mathrm{C}$.

9. Discard the column and add $20 \mu \mathrm{l}$ of $50 x \mathrm{PI}$ w/o EDTA (see Recipes; final concentration $2 x \mathrm{PI}$ w/o EDTA) to your clicked and desalted protein lysate sample.

E. Adjustment of protein concentrations 
We use a standard amido black assay as described in Dieterich et al. (2006) to determine the protein concentrations of the samples and adjust the concentration of all samples to the concentration of the sample replicate that has the lowest amount of protein with PBS-SDS pH $7.8+2 x$ PI. This adjustment improves the quality of the subsequent electrophoresis. We control the adjustment with standard silver gel staining. Other methods for the measurement of detergent-containing protein samples and subsequent adjustment are just as suitable.

F. NeutrAvidin purification

The appropriate amount of NeutrAvidin ${ }^{\mathrm{TM}}$ agarose depends on the concentration of ANL-labeled protein in your sample. If you observe a strong biotin signal in the unbound fraction of your processed samples, increase the amount of NeutrAvidin ${ }^{\mathrm{TM}}$ agarose accordingly. As a starting point, you may use $100 \mu \mathrm{l}$ NeutrAvidin ${ }^{\mathrm{TM}}$ agarose suspension for ANL-tagged proteins from a pan-neuronal driver line and $150 \mu \mathrm{l}$ suspension for ANL- labeled glial or muscle protein.

1. Gently stir the NeutrAvidin ${ }^{\mathrm{TM}}$ agarose suspension several times until the beads at the bottom are mixed uniformly within the storage buffer.

2. Transfer the appropriate amount of NeutrAvidin ${ }^{\mathrm{TM}}$ agarose suspension using a cropped pipette tip into a $1.5 \mathrm{ml}$ tube.

3. Equilibrate the NeutrAvidin ${ }^{\mathrm{TM}}$ agarose
a. Add $1 \mathrm{ml}$ PBS-Igepal-630 (see Recipes) and invert the tube several times.
b. Centrifuge at $3,000 \times g$ for $5 \mathrm{~min}$ at $4^{\circ} \mathrm{C}$.
c. Discard the supernatant carefully either with a pipette or a syringe.
d. Repeat Steps F3a-F3c twice.

4. Simultaneously incubate the desalted, clicked protein lysate with Igepal-630 (final Igepal-630 concentration in the sample: $1 \%$ ) for at least $20 \mathrm{~min}$ at $4{ }^{\circ} \mathrm{C}$ in an end over end mixer.

5. Transfer 20-40 $\mu \mathrm{l}$ of the protein lysate to a new $1.5 \mathrm{ml}$ tube (= input fraction) for SDS-PAGE and Western blot analyses, prepare the samples for SDS-PAGE as is custom in your lab and freeze the sample at $-20^{\circ} \mathrm{C}$.

6. Add the protein lysate to the equilibrated NeutrAvidin ${ }^{\mathrm{TM}}$ agarose and incubate the mixture overnight at $4{ }^{\circ} \mathrm{C}$ in an end over end mixer.

7. Centrifuge at $3,000 \times g$ for $5 \mathrm{~min}$ at $4{ }^{\circ} \mathrm{C}$.

8. Transfer supernatant into a $1.5 \mathrm{ml}$ tube (= unbound fraction). For SDS-PAGE and Western blot analyses, transfer 20-40 $\mu \mathrm{l}$ to a new $1.5 \mathrm{ml}$ tube and prepare the samples for SDS-PAGE as is custom in your lab. Freeze the sample at $-20^{\circ} \mathrm{C}$.

9. Wash NeutrAvidin ${ }^{\mathrm{TM}}$ agarose
a. Add $1 \mathrm{ml}$ PBS-Igepal-630.
b. Incubate NeutrAvidin ${ }^{\mathrm{TM}}$ agarose for $5 \mathrm{~min}$ at $\mathrm{RT}$ in an end over end mixer.
c. Centrifuge at $3,000 \times g$ for $5 \mathrm{~min}$ at $4^{\circ} \mathrm{C}$.
d. Discard supernatant.
e. Repeat Steps F9a-F9d another 4 times. 
f. Add $1 \mathrm{ml} 1 \times \mathrm{PBS} \mathrm{pH} 7.8$ to the NeutrAvidin ${ }^{\mathrm{TM}}$ agarose.

g. Incubate NeutrAvidin ${ }^{\mathrm{TM}}$ agarose for $5 \mathrm{~min}$ at RT in an end over end mixer.

h. Centrifuge at $3,000 \times g$ for $5 \mathrm{~min}$ at $4{ }^{\circ} \mathrm{C}$.

i. Discard supernatant.

j. Repeat Steps F9f-F9i twice.

10. Elution of ANL-labeled biotin-tagged proteins

a. Add 2x SDS elution buffer (see Recipes) to the tube containing the washed NeutrAvidin ${ }^{\mathrm{TM}}$ agarose.

Rule of thumb: the volume of the elution buffer should be $1 / 2$ of the NeutrAvidin ${ }^{T M}$ agarose suspension volume (that is, when you used $100 \mu$ I NeutrAvidin ${ }^{T M}$ agarose suspension, use $50 \mu$ l elution buffer).

b. Heat your samples at $95^{\circ} \mathrm{C}$ for $7 \mathrm{~min}$.

c. Cool samples down on ice for $5 \mathrm{~min}$.

d. Centrifuge at $3,000 \times g$ for $5 \mathrm{~min}$ at $4{ }^{\circ} \mathrm{C}$.

e. Transfer supernatant into a new $1.5 \mathrm{ml}$ tube (= eluate fraction).

G. SDS-PAGE and Western blot

Use a standard protocol for SDS-PAGE and Western blot. We use 5-20\% PAA, Tris-glycine gradient gels in a Hoefer Mighty Small System SE250 (Amersham Biosciences) with a constant current of $10 \mathrm{~mA}$ per gel. Separation of proteins is stopped once the bromophenol blue front leaves the separating gel. Proteins are subsequently transferred onto nitrocellulose membranes (e.g., Protran BA85, $0.45 \mu \mathrm{m}$, Whatman) at $200 \mathrm{~mA}$ for $1.5 \mathrm{~h}$. For the detection of ANL-labeled and biotin-tagged proteins use an anti-biotin antibody (e.g., rabbit-anti-biotin antibody, Bethyl Laboratories, catalog number: A150-109A). For the detection of cell-type specific candidate proteins use specific antibodies such as an anti-Dlg antibody (DSHB, USA, antibody ID 4F3 anti-discs large) for the detection of the scaffold protein Discs Large after muscular ANL-labeling, an anti-Draper antibody (DSHB, USA, antibody ID Draper 8A1) for the detection of the glial engulfment receptor Draper after glia-specific ANL-labeling or an anti-synapsin antibody (DSHB, USA, antibody ID 3C11 [anti SYNORF1]) for the detection of a synaptic protein after neuronal ANL-labeling. A sample Western blot is shown in Figure 4 depicting biotinylated ANL-harboring proteins as well as de novo synthesized synapsin in neurons. 


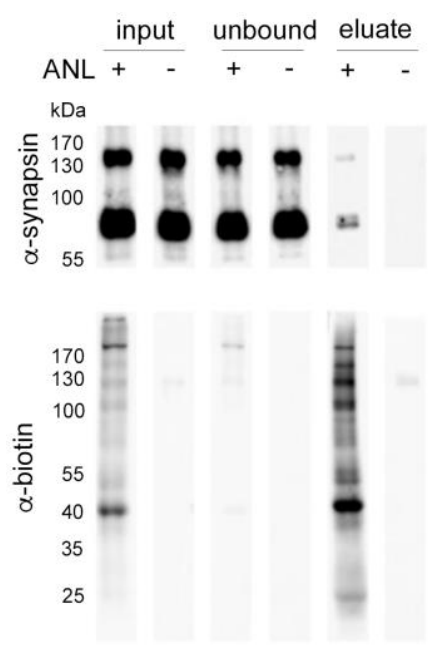

Figure 4. Western blot protein visualization and identification after chronic ANL incorporation into neuronal protein and bio-orthogonal non-canonical amino acid tagging (BONCAT). Heads from adult flies (a) chronically exposed to ANL and (b) expressing the dMetRS ${ }^{\mathrm{L262G}}$ pan-neuronally (elav ${ }^{C 155}$-Gal4, UAS-dMetRS ${ }^{\mathrm{L} 262 G_{-}}$EGFP, see our detailed bioprotocol on cell-type specific metabolic labeling of proteins with azidonorleucine in Drosophila [Erdmann et al., 2017]) were lysed (see 'protein extraction', Part II B), the ANL incorporated in the proteins was clicked to a biotin tag (see 'click chemistry', Part II C) and desalted (see 'desalting', Part II D). The protein concentration was normalized (see 'adjustment of protein concentrations', Part II E) before NeutrAvidin purification (Part II F). The representative Western blot shows the input fraction (lysate before NeutrAvidin purification), the unbound fraction (no ANL-containing proteins), and the eluate fraction (enriched ANL-labeled proteins after NeutrAvidin purification) at the global protein level (anti-biotin antibody) and for a selected candidate neuronal protein (anti-synapsin antibody). The input fraction contains all protein, that is, biotinylated and non-biotinylated protein. Therefore, the biotin signal of samples from flies exposed to ANL is diluted to approximately 1:10 depending on the respective protein compared to the same sample after NeutrAvidin purification (eluate fraction). Note that no biotin signal is observed in samples from flies not exposed to ANL, demonstrating a proper signal-to-noise ratio of the purification step. Similarly, no biotin signal is observed in the unbound fraction, demonstrating an appropriate amount of NeutrAvidin ${ }^{\mathrm{TM}}$ agarose. The relatively high synapsin signal in the unbound fraction shows that not all synapsin is biotinylated, thus that not all synapsin has ANL incorporated. This result is explained by the relatively non-invasive nature of our technique as the endogenous MetRS is not knocked out and loading methionine onto the Met-tRNA and consequently methionine into elongating polypeptide chains at the ribosome.

\section{Data analysis}

For image processing and data analysis, please see Erdmann et al. (2015). 


\section{$\underline{\text { Recipes }}$}

1. Hemolymph-like solution, HL3

$70 \mathrm{mM} \mathrm{NaCl}$

$5 \mathrm{mM} \mathrm{KCl}$

$20 \mathrm{mM} \mathrm{MgCl} 2 \cdot 6 \mathrm{H}_{2} \mathrm{O}$

$10 \mathrm{mM} \mathrm{NaHCO}_{3}$

$115 \mathrm{mM}$ sucrose

$5 \mathrm{mM}$ trehalose

5 mM HEPES

$0.1 \mathrm{mM} \mathrm{CaCl}_{2}$

Keep solution at $-20^{\circ} \mathrm{C}$ for up to 6 months

2. $4 \%$ paraformaldehyde (PFA)

$4 \mathrm{~g}$ PFA per $100 \mathrm{ml}$ 1x phosphate buffer (PB) pH 7.2

Dissolve at $60^{\circ} \mathrm{C}$, keep aliquots at $-20^{\circ} \mathrm{C}$ for up to 3 months

3. Phosphate buffer (PB) $\mathrm{pH} 7.2$
(A) $0.1 \mathrm{M} \mathrm{Na}_{2} \mathrm{HPO}_{4}, \mathrm{pH} 8.8$
(B) $0.1 \mathrm{M} \mathrm{NaH}_{2} \mathrm{PO}_{4}, \mathrm{pH} 4.1$

Add solution $B$ to solution $A$ until pH 7.2, keep at RT for up to 3 months

4. $1 \times \mathrm{PBS} \mathrm{pH} 7.4$ or 7.8

$137 \mathrm{mM} \mathrm{NaCl}$

\section{$2.7 \mathrm{mM} \mathrm{KCl}$}

$4.3 \mathrm{mM} \mathrm{Na}_{2} \mathrm{HPO}_{4}$

$1.4 \mathrm{mM} \mathrm{KH}_{2} \mathrm{HPO}_{4}$

Adjust to $\mathrm{pH} 7.4$ or 7.8 with $\mathrm{NaOH}$ and $\mathrm{HCl}$, keep at $\mathrm{RT}$ for up to 1 week

5. $\mathrm{PBT}$

$0.2 \%$ Triton $^{\circledR} \mathrm{X}-100$ in $1 \mathrm{X} \mathrm{PB} \mathrm{pH} 7.2$, keep at RT for up to 1 week

6. PBS-Tw

$1 \%(\mathrm{v} / \mathrm{v})$ Tween $^{\circledR}-20$ in $1 \mathrm{x}$ PBS $\mathrm{pH} 7.4$, keep at RT for up to 1 week

7. 50x Protease Inhibitor (PI) w/o EDTA

One 'Roche Complete EDTA-free Protease Inhibitor Cocktail' Tablet in $1 \mathrm{ml}$ ultra-pure water, store at $-20^{\circ} \mathrm{C}$ for up to 6 months

8. Homogenization buffer

$1 \times$ PBS $\mathrm{pH} 7.8$

2x PI from 50x PI stock solution

Keep the buffer on ice, prepare freshly

9. $20 \%(w / v)$ SDS

Add $2.5 \mathrm{~L} \mathrm{ddH} 2 \mathrm{O}$ to $500 \mathrm{~g}$ SDS at $60{ }^{\circ} \mathrm{C}$, stir until solved, aliquot and store at RT for up to 6 months 
10. PBS-SDS, pH 7.8

$0.05 \%$ SDS

1x PBS pH 7.8, keep at RT for up to 1 week

11. PBS-Igepal-630

$1 \%$ Igepal-630

1x PBS, pH 7.8, keep at RT for up to 1 week

12. $2 \times$ SDS elution buffer

$125 \mathrm{mM}$ Tris, $\mathrm{pH} 6.8$

$10 \% \beta$-mercaptoethanol

$2 \%$ SDS

$20 \%$ glycerol

$0.002 \%$ bromophenol blue, keep at RT for up to 3 months

\section{Acknowledgments}

This work was supported by the German Research Foundation (DFG) with an Emmy Noether Program Grant, a DIP grant and an SFB 779 grant to D.C.D., SFB 854 grants to U.T. and D.C.D., a Leibniz Society PAKT grant (LGS SynaptoGenetics) to D.C.D. and U.T., and K.M. is funded by the CBBS Postdoctoral Network Grant to K.M. and D.C.D. We thank Evelyn Dankert and Kathrin Gruss for expert technical assistance. O.K. is a member of the DFG funded Combinatorial Neurolmaging (CNI) Core Facility at LIN.

\section{Competing interests}

The authors declare no conflicts of interest or competing interests.

\section{References}

1. Agard, N. J., Prescher, J. A. and Bertozzi, C. R. (2004). A strain-promoted [3 + 2] azide-alkyne cycloaddition for covalent modification of biomolecules in living systems. J Am Chem Soc 126(46): 15046-15047.

2. Beatty, K. E., Liu, J. C., Xie, F., Dieterich, D. C., Schuman, E. M., Wang, Q. and Tirrell, D. A. (2006). Fluorescence visualization of newly synthesized proteins in mammalian cells. Angew Chem Int Ed Engl 45(44): 7364-7367.

3. Brent, J. R., Werner, K. M. and McCabe, B. D. (2009). Drosophila larval NMJ dissection. J Vis $\operatorname{Exp}(24)$ : e1107.

4. Dieterich, D. C. (2010). Chemical reporters for the illumination of protein and cell dynamics. Curr Opin Neurobiol 20(5): 623-630. 
5. Dieterich, D. C., Hodas, J. J., Gouzer, G., Shadrin, I. Y., Ngo, J. T., Triller, A., Tirrell, D. A. and Schuman, E. M. (2010). In situ visualization and dynamics of newly synthesized proteins in rat hippocampal neurons. Nat Neurosci 13(7): 897-905.

6. Dieterich, D. C., Lee, J. J., Link, A. J., Graumann, J., Tirrell, D. A. and Schuman, E. M. (2007). Labeling, detection and identification of newly synthesized proteomes with bioorthogonal noncanonical amino-acid tagging. Nat Protoc 2(3): 532-540.

7. Dieterich, D. C., Link, A. J., Graumann, J., Tirrell, D. A. and Schuman, E. M. (2006). Selective identification of newly synthesized proteins in mammalian cells using bioorthogonal noncanonical amino acid tagging (BONCAT). Proc Natl Acad Sci U S A 103(25): 9482-9487.

8. Erdmann, I., Marter, K., Kobler, O., Niehues, S., Abele, J., Müller, A., Bussmann, J., Storkebaum, E., Ziv, T., Thomas, U. and Dieterich, D. C. (2015). Cell-selective labelling of proteomes in Drosophila melanogaster. Nat Commun 6: 7521.

9. Erdmann, I., Marter, K., Kobler, O., Niehues, S., Bussmann, J., Müller, A., Abele, J., Storkebaum, E., Thomas, U. and Dieterich, D. C. (2017). Cell type-specific metabolic labeling of proteins with azidonorleucine in Drosophila. Bio-protocol 7(14): e2397.

10. Huisgen, R. (1963). Kinetics and mechanism of 1,3-dipolar cycloadditions. Angew Chem 2(11): 633-645.

11. Kho, Y., Kim, S. C., Jiang, C., Barma, D., Kwon, S. W., Cheng, J., Jaunbergs, J., Weinbaum, C., Tamanoi, F., Falck, J. and Zhao, Y. (2004). A tagging-via-substrate technology for detection and proteomics of farnesylated proteins. Proc Natl Acad Sci U S A 101(34): 12479-12484.

12. Kiick, K. L., Saxon, E., Tirrell, D. A. and Bertozzi, C. R. (2002). Incorporation of azides into recombinant proteins for chemoselective modification by the Staudinger ligation. Proc Natl Acad Sci U S A 99(1): 19-24.

13. Lallana, E., Riguera, R. and Fernandez-Megia, E. (2011). Reliable and efficient procedures for the conjugation of biomolecules through Huisgen azide-alkyne cycloadditions. Angew Chem Int Ed Eng/ 50(38): 8794-8804.

14. Laughlin, S. T., Baskin, J. M., Amacher, S. L. and Bertozzi, C. R. (2008). In vivo imaging of membrane-associated glycans in developing zebrafish. Science 320(5876): 664-667.

15. Link, A. J. and Tirrell, D. A. (2003). Cell surface labeling of Escherichia coli via copper(I)catalyzed [3+2] cycloaddition. J Am Chem Soc 125(37): 11164-11165.

16. Link, A. J., Vink, M. K., Agard, N. J., Prescher, J. A., Bertozzi, C. R. and Tirrell, D. A. (2006). Discovery of aminoacyl-tRNA synthetase activity through cell-surface display of noncanonical amino acids. Proc Natl Acad Sci U S A 103(27): 10180-10185.

17. Martin, B. R. and Cravatt, B. F. (2009). Large-scale profiling of protein palmitoylation in mammalian cells. Nat Methods 6(2): 135-138.

18. Martin, D. D., Vilas, G. L., Prescher, J. A., Rajaiah, G., Falck, J. R., Bertozzi, C. R. and Berthiaume, L. G. (2008). Rapid detection, discovery, and identification of post-translationally myristoylated proteins during apoptosis using a bio-orthogonal azidomyristate analog. FASEB J 22(3): 797-806. 
19. Massoud, T. F. and Gambhir, S. S. (2003). Molecular imaging in living subjects: seeing fundamental biological processes in a new light. Genes Dev 17(5): 545-580.

20. Müller, A., Stellmacher, A., Freitag, C. E., Landgraf, P. and Dieterich, D. C. (2015). Monitoring astrocytic proteome dynamics by cell type-specific protein labeling. PLoS One 10(12): e0145451.

21. Nguyen, K., Fazio, M., Kubota, M., Nainar, S., Feng, C., Li, X., Atwood, S. X., Bredy, T. W. and Spitale, R. C. (2017). Cell-selective bioorthogonal metabolic labeling of RNA. J Am Chem Soc 139(6): 2148-2151.

22. Niehues, S., Bussmann, J., Steffes, G., Erdmann, I., Kohrer, C., Sun, L., Wagner, M., Schafer, K., Wang, G., Koerdt, S. N., Stum, M., Jaiswal, S., RajBhandary, U. L., Thomas, U., Aberle, H., Burgess, R. W., Yang, X. L., Dieterich, D. and Storkebaum, E. (2015). Impaired protein translation in Drosophila models for Charcot-Marie-Tooth neuropathy caused by mutant tRNA synthetases. Nat Commun 6: 7520.

23. Palaniappan, K. K. and Bertozzi, C. R. (2016). Chemical glycoproteomics. Chem Rev 116(23): 14277-14306.

24. Prescher, J. A. and Bertozzi, C. R. (2005). Chemistry in living systems. Nat Chem Biol 1(1): 1321.

25. Ramachandran, P. and Budnik, V. (2010). Dissection of Drosophila larval body-wall muscles. Cold Spring Harb Protoc 2010(8): pdb prot5469.

26. Ramil, C. P. and Lin, Q. (2013). Bioorthogonal chemistry: strategies and recent developments. Chem Commun (Camb) 49(94): 11007-11022.

27. Rostovtsev, V. V., Green, L. G., Fokin, V. V. and Sharpless, K. B. (2002). A stepwise huisgen cycloaddition process: copper(I)-catalyzed regioselective "ligation" of azides and terminal alkynes. Angew Chem Int Ed Engl 41(14): 2596-2599.

28. Saxon, E. and Bertozzi, C. R. (2000). Cell surface engineering by a modified Staudinger reaction. Science 287(5460): 2007-2010.

29. Seo, T. S., Bai, X., Ruparel, H., Li, Z., Turro, N. J. and Ju, J. (2004). Photocleavable fluorescent nucleotides for DNA sequencing on a chip constructed by site-specific coupling chemistry. Proc Natl Acad Sci U S A 101(15): 5488-5493.

30. tom Dieck, S., Kochen, L., Hanus, C., Heumuller, M., Bartnik, I., Nassim-Assir, B., Merk, K., Mosler, T., Garg, S., Bunse, S., Tirrell, D. A. and Schuman, E. M. (2015). Direct visualization of newly synthesized target proteins in situ. Nat Methods 12(5): 411-414.

31. Tornoe, C. W., Christensen, C. and Meldal, M. (2002). Peptidotriazoles on solid phase: [1,2,3]triazoles by regiospecific copper(i)-catalyzed 1,3-dipolar cycloadditions of terminal alkynes to azides. J Org Chem 67(9): 3057-3064.

32. Tsien, R. Y. (1998). The green fluorescent protein. Annu Rev Biochem 67: 509-544.

33. Wang, Q., Chan, T. R., Hilgraf, R., Fokin, V. V., Sharpless, K. B. and Finn, M. G. (2003). Bioconjugation by copper(I)-catalyzed azide-alkyne [3+2] cycloaddition. J Am Chem Soc 125(11): 3192-3193. 